\title{
Efecto de acondicionadores naturales y sintéticos sobre los cationes solubles y la infiltración del agua en un Aridisol(1)
}

\author{
Manuel Henríquez ${ }^{(2)}$, Orlando Rodríguez ${ }^{(2)}$, Francisco Montero(2) $^{(2)}$ \\ y Alexander Hernández ${ }^{(3)}$
}

\begin{abstract}
Resumen - El objetivo del trabajo fue evaluar el efecto de diversos acondicionadores sobre los cationes solubles y la infiltración del agua en un suelo Vertic Haplocambid, arcilloso muy fino, de la depresión de Quibor, Venezuela. Fueron aplicadas soluciones de acondicionadores naturales de cardón dato (Lemaireocereus griceus (Haw.) Br. \& Rose) y de cardón lefaria (Cereus deficiens Otto \& Dietr), a concentraciones de 2.000, 1.000 y $500 \mathrm{mg} \mathrm{L}^{-1}$, solución de un acondicionador sintético, la poliacrilamida, en concentración de $10 \mathrm{mg} \mathrm{L}^{-1}$ y agua del acueducto local. Las soluciones de cada uno de esos tratamientos fueron vertidas sobre mini canales rellenos del suelo, para simular el flujo del agua en surcos. Los acondicionadores naturales incrementaron la infiltración y produjeron una relación Ca-Mg-Na en el suelo, mas adecuada que la producida con la aplicación de la poliacrilamida o el agua sin aditivos.
\end{abstract}

Términos para indexación: Lemaireocereus griceus, Cereus deficiens, poliacrilamida, rehabilitación de tierras.

Effect of natural and synthetic conditioners on soluble cations and water infiltration in an Aridisol

\begin{abstract}
The objective of this work was to evaluate the effect of several conditioners on soluble cations and water infiltration on a fine clay Vertic Haplocambid soil collected on the Quibor depression, Venezuela. Solutions of two natural conditioners from dato cactus (Lemaireocereus griceus (Haw.) Br. \& Rose) and lefaria cactus (Cereus deficiens Otto \& Dietr), on concentrations of 2,000, 1,000 and $500 \mathrm{mg} \mathrm{L}^{-1}$, a synthetic polyacrilamide conditioner solution of $10 \mathrm{mg} \mathrm{L}^{-1}$ and local tap water were applied to the soil. All solutions and the tap water were poured in a mini flume to simulate the irrigation process on furrows. The natural conditioners increased the infiltration and produced a better $\mathrm{Ca}-\mathrm{Mg}-\mathrm{Na}$ relationship as compared with that produced by the polyacrilamide and the tap water without additives.
\end{abstract}

Index terms: Lemaireocereus griceus, Cereus deficiens, polyacrilamide, reclamation.

\section{Introducción}

El avance del agua en surcos produce un flujo turbulento con alto poder erosivo, lo cual contribuye al arrastre del suelo y su posterior sedimentación donde el agua reduce la velocidad, formándose una

\footnotetext{
(1) Aceptado para publicación en 2 de octubre de 2002. Trabajo financiado por el Consejo de Desarrollo Científico, Humanístico y Tecnológico de la Universidad Centroccidental Lisandro Alvarado (Ucla), Lara, Venezuela.

(2) Ucla, Decanato de Agronomía, Dep. de Química y Suelos, Apartado 400, Lara, Venezuela. E-mail: mhenriq@reacciun.ve, orodrigu@telcel.net.ve

(3) Ucla, Decanato de Agronomía, Dep. de Ciencias Biológicas. E-mail: alexher55@hotmail.com
}

capa de estructura laminar o sello superficial, el cual reduce la permeabilidad del suelo al agua y al aire, generando un ambiente anaeróbico en la zona radical, que puede traducirse en reducción de la productividad (Lentz et al., 1992).

Shainberg \& Rodríguez (1993), reportaron que en los suelos de la depresión de Quibor en Lara, Venezuela, la estructura es inestable y que el sellado superficial es el mecanismo más determinante de los bajos rendimientos de los cultivos. Shainberg \& Rodríguez (1993) y Ramírez et al. (1999), demostraron que estos suelos son muy dispersivos. Ramírez et al. (1999) reportaron que en los suelos de Quibor, la presencia de una costra en la superficie de estos suelos reduce la tasa de infiltración, pero cuando ese sello superficial es formado por arcillas 
floculadas, se genera una estructura más permeable, la cual permite una mayor tasa de infiltración y que el frente de agua alcance mayor profundidad. La disposición de las sales a mayor profundidad es sumamente ventajosa para los cultivos (Emerson, 1984).

Rodríguez (1982) y Rodríguez \& Perkins (1984) señalan que el Ca es el catión más abundante en el complejo de cambio y en la solución de este suelo y que el mineral más abundante de la fracción arcilla es la ilita y en menor abundancia se consiguen la pirofilita, la montmorillonita y la calcita. Pérez et al. (1992, 1995) y Villafañe et al. (1999) reportan que el complejo de cambio de estos suelos tiene altos contenidos de $\mathrm{Ca}$ y $\mathrm{Mg}$ y un menor contenido de sodio.

La dispersividad de las arcillas y los problemas de índole física que este fenómeno causa a los suelos han sido atribuidos a varios factores, entre los cuales destacan el tipo de arcilla, el tipo de catión presente en el complejo de cambio y en la solución del suelo (Ramírez et al., 1999). Shainberg et al. (1990) indican que las arcillas montmorillonita e ilita, le confieren características dispersivas a los suelos. Emerson (1984) señala que un aumento en los iónes Mg y Na, así como una disminución de los iónes $\mathrm{Ca}$ en el complejo de cambio, aumentan la dispersividad del suelo, explicando esos procesos debido a que tanto el $\mathrm{Na}$ como el Mg tienen una energía de hidratación mayor que la del $\mathrm{Ca}$, de modo que el grado de disociación del $\mathrm{Na}$ y del $\mathrm{Mg}$ en la superficie de las arcillas es mayor al del $\mathrm{Ca}$ y en consecuencia, esto permite el incremento de las fuerzas repulsivas entre las partículas de arcilla.

Para reducir estos problemas, normalmente se han utilizado técnicas de cobertura y de incorporación de materia orgánica. Mas recientemente, se ha venido extendiendo el uso de acondicionadores, con el fin de estabilizar la estructura y mejorar la porosidad y la infiltración del agua en el suelo (Lentz et al., 1992). Estos acondicionadores pueden ser naturales o sintéticos. Entre los naturales, son conocidos los polímeros tipo ácidos poliurónicos, los ácidos algínicos, los polisacáridos y el humus. De los polímeros sintéticos, el más conocido es la poliacrilamida (PAM), un polímero de alto peso molecular, que al aplicarse disuelto en el agua de riego, aumenta las fuerzas cohesivas entre las partí- culas del suelo (Shainberg et al., 1990). La efectividad de la PAM como acondicionador ha sido demostrada por Shainberg et al. (1990) y por Bouranis et al. (1995). Shainberg et al. (1990) sugieren que el mecanismo de funcionamiento de la PAM se explica a través de dos tipos de puentes partículas-cationes-polímeros, los cuales son los responsables de la adsorción y enlace entre las partículas de arcilla, las cuales llegan a flocular y a formar agregados. Estos dos tipos de puentes conducen a una mayor estabilidad de los agregados al agua, haciendo que los suelos sean más permeables y en consecuencia, la precolación y la profundidad de penetración del agua en el suelo, sea mayor (Tisdall \& Oades, 1982).

En este trabajo, la PAM será utilizada como material de referencia, para establecer las concentraciones de los acondicionadores naturales (AN), que produzcan un efecto similar a las dosis ya probadas de PAM.

Los extractos de Opuntia ficus-indica (Linaeus) han sido utilizados por Gardiner et al. (1999) y los polímeros de Cyamopsis tragonoloba L. (Taub), por Wallace (1986a, 1986b), para mejorar las propiedades físicas del suelo. Henríquez et al. (1999a, 1999b) y Montero (1999) utilizaron el parénquima de Lemaireocereus griceus (Haw.) (Br. \& Rose) o cardón dato (CD), y de Cereus deficiens (Otto \& Dietr) o cardón lefaria (CL), consiguiendo una reducción de la erosión en el riego por surcos, incremento de la permeabilidad y de la macroporosidad del mismo suelo bajo estudio.

Las Opuntia sp. son plantas suculentas que contienen células parenquimáticas normalmente grandes y de pared fina, que tienen una delgada capa de citoplasma, la cual carece de cloroplastos o contiene unos pocos, en los cuales se almacena agua y contienen también una gran vacuola, con jugo celular de carácter mucilaginoso similar a las pectinas (Gardiner et al., 1999). Henríquez et al. (1999a, 1999b) reportaron que el CD y el CL que utilizaron contienen gran cantidad de mucílago, lo cual les confiere características similares a las reportadas para las Opuntia sp. El cardón dato es una Cactacea con corteza de color verde oscuro, produce un tronco central de no más de $50 \mathrm{~cm}$ de altura, con 5 a 8 espinas en cada cojincillo y sin espina central, su fruto es esférico, rojo y con espinas que pierde al madurar 
(Smith, 1975). El cardón lefaria también es una Cactacea, su corteza es de color grisáceo, produce un tronco central hasta con más de $1 \mathrm{~m}$ de altura, con numerosas espinas en cada cojincillo y con espina central, su fruto es ovalado, verdoso, sin espinas (Smith, 1975).

Los mucílagos son hidratos de carbono con abundancia de polisacáridos en cuya estructura están presentes los grupos - $\mathrm{NH}_{2},-\mathrm{COOH}$ y -OH entre otros. Estos grupos al disociarse en presencia de agua dejan cargas negativas libres, las cuales funcionan como puentes o agentes de enlace directo con las arcillas, uniendo las cargas negativas de los polisacáridos con las cargas positivas de las arcillas (Bolan et al., 1999). Según Gardiner et al. (1999), el fenómeno que permite explicar el funcionamiento de los AN es similar al de la PAM, reportando que la estructura del mucílago utilizado en sus experimentos era similar al de las pectinas y el de estas, similar al de la PAM. Estos mucílagos inducen floculación y agregación de las partículas del suelo, haciendo que estas precipiten debido a su alto peso molecular.

Otro factor que incrementa la floculación de las arcillas es el incremento de la concentración de sales en la solución del suelo (Shainberg et al., 1990).

Debido a la inestabilidad estructural y a la salinidad de los suelos de la depresión de Quibor, se han generado problemas como la reducción de la permeabilidad y la disminución de su productividad agrícola (Pérez et al., 1992, 1995; Villafañe et al., 1999).

El objetivo del trabajo fue evaluar el efecto de diversos acondicionadores sobre los cationes solubles y la infiltración del agua en un suelo Vertic Haplocambid.

\section{Material y Métodos}

El experimento fue realizado en el laboratorio de investigación del Departamento de Química y Suelos del Decanato de Agronomía de la Universidad Centroccidental Lisandro Alvarado en Cabudare, E. Lara, Venezuela.

Al CD y al CL, se les despojó de la corteza y la médula, para obtener el parénquima acuoso, el cual fue desmenuzado en una licuadora hasta lograr un tamaño de partículas muy fino. El material obtenido fue diluido utilizando agua del acueducto local con conductividad eléctrica (CE), de $0,7 \mathrm{dS} \mathrm{m}^{-1}$, para preparar soluciones con concentraciones de $2.000,1.000$ y $500 \mathrm{mg} \mathrm{L}^{-1}$ de cada cardón. Una vez preparadas estas soluciones, se les midió nuevamente su CE. Adicionalmente con el mismo tipo de agua fue preparada una solución a concentración de $10 \mathrm{mg} \mathrm{L}^{-1}$ de una poliacrilamida (PAM) de tipo aniónico de alto peso molecular $\left(10^{7}\right)$ y de baja densidad de carga $(20 \%$ de hidrólisis), comercializada en Venezuela con el nombre de Magnifloc 836 (Francisco et al., 1994). Como testigo se utilizó agua proveniente de las tuberías del acueducto local con CE de $0,7 \mathrm{dS} \mathrm{m}^{-1}$.

El suelo utilizado fue tomado en la finca la Caimana, ubicada en el sector denominado Ojo de Agua Abajo, en Quibor, E. Lara. Según Pérez et al. (1995), el perfil representativo de estos suelos (perfil № 11) clasificó como un Vertic Haplocambid, arcilloso muy fino, mixto, no ácido, isohipertérmico. Los análisis químicos del perfil para los primeros $27 \mathrm{~cm}$ reportan una relación de adsorción de sodio (RAS) de $15,49 \mathrm{cmol}_{\mathrm{c}} \mathrm{L}^{-1}$ y entre los 27 y $72 \mathrm{~cm}$, una RAS de $4,13 \mathrm{cmol}_{\mathrm{c}} \mathrm{L}^{-1}$. Más del $90 \%$ de la capacidad de intercambio de cationes (CIC) reportada para los dos primeros horizontes corresponde a $\mathrm{Ca}$ y $\mathrm{Mg}$, la $\mathrm{CE}$ reportada para los mismos horizontes es mayor a $4 \mathrm{dS} \mathrm{m}^{-1}$. Muestras de los primeros $20 \mathrm{~cm}$ del suelo mencionado fueron llevadas al laboratorio.

Fueron fabricados unos mini canales de metal, formados por un depósito para suelo de $50 \mathrm{~cm}$ de largo, $12 \mathrm{~cm}$ de ancho y $7 \mathrm{~cm}$ de alto, al cual le fue soldado en los extremos del lado largo, dos extensiones en forma de $\mathrm{V}$, de $50 \mathrm{~cm}$ cada uno. Los depósitos de estos mini canales fueron rellenados con el suelo, para aplicarle posteriormente los tratamientos y el agua del acueducto. Las soluciones preparadas a partir del CD, CL y la PAM, fueron vertidas sobre uno de los extremos del mini canal, simulando el flujo del agua de riego en los surcos y recogidas para análisis en el otro extremo. Cada una de estas pruebas fueron repetidas en tres canaletas. La profundidad de penetración del agua fue determinada midiendo la profundidad del frente de agua en tres puntos diferentes en cada canaleta.

Para determinar el efecto de las diferentes concentraciones de CD, CL, PAM y el agua natural, fueron preparadas pastas con $400 \mathrm{~g}$ del suelo, saturándolas con las soluciones y concentraciones previamente descritas, así como con el agua natural. Las pastas saturadas fueron colocadas en embudos Buchner conectados a una bomba de vacío, para luego determinarles la CE y los cationes solubles en el extracto.

Los resultados fueron analizados mediante la prueba de medias con un modelo totalmente aleatorizado con tres repeticiones, al $5 \%$ de probabilidad por la prueba de rangos múltiples de Duncan. 


\section{Resultados y Discusión}

La CE del agua a la cual se le agregaron los AN, para preparar las diversas soluciones utilizadas, aumentó desde el valor original de $0,7 \mathrm{dS} \mathrm{m}^{-1}$ a valores entre 1,3 y $1,86 \mathrm{dS} \mathrm{m}^{-1}$, como consecuencia de la disolución de los AN. Estos valores obtenidos, superaron el valor crítico de floculación reportado para ese suelo por Montero (1999). La CE del agua a la cual se le aplicó la PAM no fue modificada.

La penetración del agua en el suelo resultó significativamente mayor con el uso de los AN y la PAM que con el agua natural utilizada como testigo (Cuadro 1). Los tratamientos con CD incrementaron significativamente la profundidad alcanzada por el agua con relación a los tratamientos con CL, la PAM y el testigo. No existieron diferencias significativas entre los tratamientos con CD. De acuerdo a estos resultados, se sugiere que el uso del CD 500, el AN de menor concentración y de los más eficaces entre los AN probados, resulta el más conveniente desde el punto de vista económico, para mejorar la penetración de agua.

Se sugiere que la mayor efectividad del CD en promover la infiltración profunda podría explicarse sobre la base que los polímeros que conforman su mucílago, tienen una alta densidad de carga y un alto peso molecular, sugerencia que ha sido adelantada también por Gardiner et al. (1999). La concordancia entre el presente trabajo y el de
Gardiner et al. (1999) permite sugerir que la estructura del mucílago contenido en el tejido parenquimático de los AN utilizados sería similar al de las pectinas.

En consecuencia, se sugiere que tal como las pectinas, el mucílago de los AN probados contendría grupos hidroxilos y carboxilos en su estructura, los cuales al disociarse, al igual que la PAM, dejarían cargas negativas libres, promoverían la floculación de las partículas y por ende, aumentarían la posibilidad de percolación del agua a mayores profundidades (Tisdall \& Oades, 1982; Shainberg et al., 1990).

El efecto de los AN sobre la mayor profundidad de penetración del agua en el suelo también podría ser atribuible al aumento de la concentración de electrolitos en el agua (Cuadro 1), efecto demostrado por Ramírez et al. (1999), quienes reportan que el incremento de la CE del agua aplicada a estos suelos favoreció la formación de un sello deposicional de estructura más permeable.

Todos los tratamientos con CD y CL produjeron valores estadísticamente menores de Ca en el extracto que los obtenidos con la PAM y con el testigo. Este resultado indica que los $\mathrm{AN}$ causaron una menor movilización de Ca hacia el extracto de saturación que la PAM y el agua de acueducto utilizada como testigo (Cuadro 1).

La menor concentración de Ca en el extracto de saturación donde se aplicaron los AN (Cuadro 1), pudiera explicarse sobre la base que estos AN

Cuadro 1. Penetración del agua en el suelo, conductividad eléctrica (CE), concentración de cationes y relaciones Ca-Mg$\mathrm{Na}$ en el extracto de saturación de un suelo tratado con los acondicionadores naturales cardón dato (CD), cardón lefaria (CL) y la poliacrilamida (PAM) $)^{(1)}$.

\begin{tabular}{|c|c|c|c|c|c|c|c|c|c|c|}
\hline \multirow{3}{*}{$\begin{array}{l}\text { Concentración } \\
\left(\mathrm{mg} \mathrm{L}^{-1}\right)\end{array}$} & \multirow{3}{*}{$\begin{array}{l}\text { Profundidad de } \\
\text { penetración del } \\
\text { agua }(\mathrm{cm})\end{array}$} & \multirow{3}{*}{ 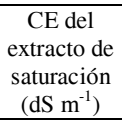 } & \multicolumn{4}{|c|}{ Cationes extraídos } & \multirow{3}{*}{$\begin{array}{l}\text { Cationes } \\
\text { totales } \\
\text { extraídos } \\
\left(\mathrm{cmol}_{\mathrm{c}} \mathrm{L}^{-1}\right)\end{array}$} & \multirow{2}{*}{\multicolumn{3}{|c|}{$\begin{array}{c}\text { Relación en el extracto } \\
\text { de saturación }\end{array}$}} \\
\hline & & & \multirow{2}{*}{$\mathrm{K}^{+}$} & \multirow{2}{*}{\multicolumn{2}{|c|}{$\mathrm{Ca}^{2+} \quad \mathrm{Mg}^{2+}$}} & \multirow{2}{*}{$\mathrm{Na}^{+}$} & & & & \\
\hline & & & & & & & & $\mathrm{Ca} / \mathrm{Na}$ & $\mathrm{Mg} / \mathrm{Na}$ & $\mathrm{Mg} / \mathrm{Ca}$ \\
\hline & \multicolumn{10}{|c|}{ Cardón dato } \\
\hline 2.000 & $4,457 \mathrm{a}$ & $5,067 \mathrm{~d}$ & $0,342 \mathrm{c}$ & $27,19 \mathrm{c}$ & $12,18 \mathrm{~b}$ & $18,73 b$ & $58,45 \mathrm{c}$ & $1,48 \mathrm{cde}$ & $0,65 \mathrm{ab}$ & $0,45 \mathrm{ab}$ \\
\hline 1.000 & $4,407 \mathrm{a}$ & $6,200 c$ & $0,466 \mathrm{a}$ & $32,50 \mathrm{~b}$ & $15,54 \mathrm{a}$ & $23,80 \mathrm{a}$ & $72,31 \mathrm{ab}$ & $1,36 \mathrm{de}$ & $0,65 \mathrm{ab}$ & $0,48 \mathrm{a}$ \\
\hline \multirow[t]{2}{*}{500} & $4,387 \mathrm{a}$ & $6,257 \mathrm{bc}$ & $0,455 \mathrm{a}$ & $32,20 \mathrm{~b}$ & $13,75 \mathrm{ab}$ & $23,80 \mathrm{a}$ & $70,21 \mathrm{ab}$ & $1,35 \mathrm{e}$ & $0,58 \mathrm{~b}$ & $0,43 \mathrm{ab}$ \\
\hline & \multicolumn{10}{|c|}{ Cardón lefaria } \\
\hline 2.000 & $3,543 b$ & $6,200 c$ & $0,416 \mathrm{ab}$ & $31,24 \mathrm{~b}$ & $14,00 \mathrm{ab}$ & $20,50 \mathrm{ab}$ & $66,15 b c$ & $1,52 \mathrm{cde}$ & $0,68 \mathrm{a}$ & $0,46 \mathrm{ab}$ \\
\hline 1.000 & $3,400 \mathrm{bc}$ & $6,133 \mathrm{c}$ & $0,387 \mathrm{bc}$ & $31,29 \mathrm{~b}$ & $13,73 \mathrm{ab}$ & $20,50 \mathrm{ab}$ & $66,11 b c$ & $1,53 \mathrm{~cd}$ & $0,66 \mathrm{a}$ & $0,44 a b$ \\
\hline \multirow[t]{2}{*}{500} & $3,133 \mathrm{c}$ & $6,200 \mathrm{c}$ & $0,394 b c$ & $32,63 b$ & $13,80 \mathrm{ab}$ & $21,03 \mathrm{ab}$ & $64,53 \mathrm{bc}$ & $1,55 \mathrm{c}$ & $0,65 \mathrm{ab}$ & $0,42 b$ \\
\hline & \multicolumn{10}{|c|}{ Poliacrilamida } \\
\hline \multirow[t]{2}{*}{10} & $3,633 b$ & $7,203 \mathrm{a}$ & $0,473 \mathrm{a}$ & $42,42 \mathrm{a}$ & $14,53 \mathrm{ab}$ & $21,43 \mathrm{ab}$ & $78,64 \mathrm{a}$ & $1,97 \mathrm{a}$ & $0,68 \mathrm{a}$ & $0,34 \mathrm{c}$ \\
\hline & \multicolumn{10}{|c|}{ Agua } \\
\hline 0 & $2,733 \mathrm{~d}$ & $7,000 \mathrm{ab}$ & $0,393 b c$ & $40,68 \mathrm{a}$ & $14,31 \mathrm{ab}$ & $22,77 \mathrm{ab}$ & $78,15 \mathrm{a}$ & $1,77 \mathrm{~b}$ & $0,62 b$ & $0,35 \mathrm{c}$ \\
\hline $\mathrm{CV}(\%)$ & 5,7 & 6,96 & 5,99 & 5,00 & 10,18 & 10,32 & 6,75 & 5,7 & 6,27 & 7,23 \\
\hline
\end{tabular}


tuvieren en la estructura del mucílago grupos funcionales como los $-\mathrm{COOH},-\mathrm{NH}_{4} \mathrm{y}-\mathrm{OH}$, los cuales al disociarse y unirse con cargas positivas de las arcillas pudieren haber aumentado la capacidad de retener cationes (CIC) por el suelo. La extracción de $\mathrm{Mg}$ y de Na por los AN resultó estadísticamente similar a la de la PAM y el testigo (Cuadro 1).

La extracción de $\mathrm{K}$ es mucho menor que la de los otros elementos y no muestra una tendencia diferencial entre todos los tratamientos probados (Cuadro 1). La menor extracción de $\mathrm{K}$ pudiera explicarse porque en estos suelos hay ilita, mineral que retiene con mayor fuerza al $\mathrm{K}$ que a los otros principales cationes de intercambio.

El CD 2.000 y todos los tratamientos con CL redujeron el lavado de cationes totales hacia el extracto, con relación a la PAM y el agua natural (Cuadro 1). Esto significaría que estos AN tenderían a reducir el lavado de sales, debido a que los cationes adsorbidos en sus enlaces funcionales serían retenidos con más fuerza en el complejo suelo-AN-catión.

Los valores de $\mathrm{Ca}, \mathrm{Mg}$ y de cationes totales en el extracto de saturación obtenidos con la PAM y el testigo resultaron similares (Cuadro 1). Esta situación puede explicarse sobre la base que la PAM, en la concentración probada, no logra incrementar de manera significativa la retención de cationes en el complejo de cambio del suelo utilizado.

La relación $\mathrm{Ca} / \mathrm{Na}$ en el extracto resultó significativamente menor para todos los AN que la de la PAM y el testigo (Cuadro 1). Un menor valor de la relación $\mathrm{Ca} / \mathrm{Na}$ en el extracto indica que fue extraído más $\mathrm{Na}$ que Ca por los AN que el extraído por la PAM y por el testigo. La eliminación preferencial del complejo de cambio del catión $\mathrm{Na}$ en presencia de $\mathrm{Ca}$ resulta beneficioso en la recuperación de suelos dispersivos afectados por sodicidad (Emerson, 1984).

La relación $\mathrm{Mg} / \mathrm{Ca}$ en el extracto fue estadísticamente mayor en los AN que en la PAM y el testigo, esto indica una extracción de $\mathrm{Mg}$ superior a la de $\mathrm{Ca}$ en los tratamientos con los AN (Cuadro 1). Emerson (1984), reporta que el efecto dispersivo del Mg puede ser parecido al del sodio. Con respecto a la relación $\mathrm{Mg} / \mathrm{Ca}$, la aplicación de AN favorece estos suelos, debido a que extraen una mayor proporción de $\mathrm{Mg}$ que de calcio.
La mayor extracción de Ca y la menor extracción de $\mathrm{Mg}$ y de Na por parte de la PAM y el agua natural traerían como consecuencia el aumento de las relaciones $\mathrm{Mg} / \mathrm{Ca}$ y $\mathrm{Na} / \mathrm{Ca}$ en el suelo (Cuadro 1), lo cual podría incrementar su dispersividad. Se sugiere que en el manejo de estos suelos debe ponerse atención a las enmiendas y prácticas, que pudiesen incrementar esas relaciones en el complejo de cambio.

El uso de los AN resultó apropiado en este suelo, porque produjo una relación adecuada entre los iónes $\mathrm{Ca}, \mathrm{Mg}$ y $\mathrm{Na}$ y en consecuencia, promovería el fortalecimiento de los enlaces entre las partículas de arcilla, reduciendo la dispersión.

Como el efecto del CD 500 fue el que combinó la más conveniente profundidad de penetración del agua y la menor y más conveniente relación $\mathrm{Ca} / \mathrm{Na}$ en el extracto, se sugiere que entre los AN probados, la dosis de CD 500 es la más recomendable.

\section{Conclusiones}

1. El tratamiento del suelo con suspensiones de acondicionadores naturales es benéfico, porque aumenta la profundidad de penetración del agua.

2. Los efectos más relevantes con relación a la profundidad de penetración del agua y las relaciones entre los cationes en el extracto son obtenidas por el tratamiento con suspensiones de cardón dato en la dosis de $500 \mathrm{mg} \mathrm{L}^{-1}$.

3. Las relaciones $\mathrm{Ca} / \mathrm{Na}$ y $\mathrm{Mg} / \mathrm{Ca}$ más adecuadas en el extracto de saturación del suelo, son obtenidas con el uso de los acondicionadores naturales.

\section{Referencias}

BOLAN, N. S.; NAIDU, R.; SYERA, J. K.; TILLMAN, R. W. Surface charge and solute interactions. Advances in Agronomy, Indianapolis, v. 67, n. 1, p. 87-140, 1999.

BOURANIS, D. L.; THEODOROPOULOS, A. G.; DROSSOPOULOS, J. B. Designing synthetic polymers as soil conditioners. Communications in Soil Science and Plant Analysis, Monticello, v. 26, n. 13/14, p. 14551480, 1995.

EMERSON, W. Soil salinity under irrigation: processes and management. Berlin: Springer, 1984. 432 p.

FRANCISCO, D.; RODRÍGUEZ, O.; ORTIZ, N. Efecto de poliacrilamidas aniónicas en los sedimentos de agua de 
riego y la emergencia de cebolla (Allium cepa $\mathrm{L}$.). Suelos Ecuatoriales, Bogotá, v. 24, n. 1, p. 84-87, 1994.

GARDINER, D.; PETER, F.; CARR, T. Cactus extract increases water infiltration rates in two soils. Communications in Soil Science and Plant Analysis, Monticello, v. 130, n. 15/16, p. 1707-1712, 1999.

HENRÍQUEZ, M.; MONTERO, F.; RODRÍGUEZ, O.; HERNÁNDEZ, A. Efecto de acondicionadores naturales sobre la floculación, la conductividad hidráulica y la penetración del agua en el perfil. In: CONGRESO LATINOAMERICANO DE LA CIENCIA DEL SUELO, 14., 1999, Pucón. Memorias... Pucón: Sociedad Chilena de la Ciencia del Suelo, 1999a. p. 4-10.

HENRÍQUEZ, M.; RODRÍGUEZ, O.; MONTERO, F.; HERNÁNDEZ, A. Efecto de acondicionadores naturales y poliacrilamidas aniónicas (PAM) sobre algunas propiedades físicas y químicas de un suelo de Quíbor. In: CONGRESO VENEZOLANO DE LA CIENCIA DEL SUELO, 15., 1999, Barquisimeto. Memorias... Barquisimeto: Sociedad Venezolana de la Ciencia del Suelo, 1999b. p. 220-224.

LENTZ, R.; SHAINBERG, I.; SOJKA, R.; CARTER, D. Preventing irrigation furrow erosion with small applications of polymers. Soil Science Society of America Journal, Madison, v. 56, n. 6, p. 1926-1932, 1992.

MONTERO, F. Efecto de la aplicación de acondicionadores naturales sobre algunas propiedades físicas de un suelo de Quibor, E. Lara. 1999. 33 p. Trabajo (Técnico Superior en Ciencia Agrícola) - Universidad Centroccidental Lisandro Alvarado, Carora. 1999.

PÉREZ, J. R.; GÓMEZ, J.; GUÉDEZ, J.; OHEP, C.; MARCANO, F.; FRANCISCO, D. Estudio semidetallado de clasificación de tierras con fines de riego del valle de Quibor. Barquisimeto: Sistema Hidráulico Yacambú Quibor, 1992. 193 p.

PÉREZ, J. R.; SCHARGEL, R.; GÓMEZ, J.; OHEP, C. Estudio semidetallado de suelos a nivel de serie del Valle de Quibor. Barquisimeto: Sistema Hidráulico Yacambú Quibor, 1995. 78 p.
RAMÍREZ, H.; RODRÍGUEZ, O.; SHAINBERG, I. Effect of gypsum on furrow erosion and intake rate. Soil Science, Baltimore, v. 164, n. 3, p. 351-357, 1999.

RODRÍGUEZ, O. Mineralogy and related properties of selected soils on Lara landscapes. 1982. 63 p. Dissertation (M.Sc. in Agriculture) - University of Georgia, Athens, 1982.

RODRÍGUEZ, O.; PERKINS, H. F. Turbio soils of Northwestern Venezuela. Properties and placement in ustropeptic subgroup. Soil Science, Baltimore, v. 138, n. 1 , p. 33-39, 1984

SHAINBERG, I.; RODRÍGUEZ, O. Reporte sobre la gira de estudio al valle de Quibor. Barquisimeto: Sistema Hidráulico Yacambú Quibor, 1993. 45 p.

SHAINBERG, I.; WARRINGTON, D. N.; RENGASSAMY, P. Water quality and PAM interactions in reducing surface sealing. Soil Science, Baltimore, v. 149, n. 3 , p. 301-307, 1990.

SMITH, R. Ecología de las plantas leñosas del espinar de los estados Lara y Falcón de Venezuela y clave ilustrada en base a sus características vegetativas. Acta Botánica de Venezuela, Caracas, v. 10, n. 1, p. 87-129, 1975.

TISDALL, J. M.; OADES, J. M. Organic matter and water-stables-aggregates in soils. Journal of Soil Science, Baltimore, v. 33, n. 2, p. 141-163, 1982.

VILLAFAÑE, R.; AZPURUA, M.; ABARCA, O.; RUIZ, $T$. Determinación de la variabilidad espacial de las sales en el Valle de Quibor. Barquisimeto: Sistema Hidráulico Yacambú Quibor/Empresa Rental de la Facultad de Agronomía, 1999. 62 p.

WALLACE, A. A polysaccharide (Guar) as a soil conditioner. Soil Science, Baltimore, v. 141, n. 3, p. 371373, 1986a.

WALLACE, A. Effect of polymers in solution culture on growth and mineral composition of tomatoes. Soil Science, Baltimore, v. 141, n. 3, p. 395-396, 1986 b. 